\title{
Algumas recomendações para atletas jovens corredores de 400 metros com problemas no sistema cardiovascular.
}

\section{Algunas recomendaciones para atletas jóvenes corredores de 400 metros planos con problemas en el sistema cardiovascular.}

\author{
Gabriela Alexandra Villalba Garzón. ${ }^{1}$, Soledad Ríos Tapia. ${ }^{2}$, Vladimir Quizhpe \\ Luzuriaga. $^{3} \&$ Luis Rafael Valverde Jumbo. ${ }^{4}$
}

\section{DOI: https://doi.org/10.33262/anatomiadigital.v4i2.1616}

\section{Resumo.}

A corrida de 400 metros em todos os eventos de atletismo há muito tempo é um ponto de atração devido às características biológicas que são realizadas neste desporte. Por sua vez, esta tem gerado muito interesse por parte dos treinadores que buscam diversos recursos para conhecer os fatores limitantes do desempenho bem como as características cardiovasculares mais importantes deste disciplina. Com o objetivo de: Descrever os efeitos das sobrecargas de treinamento

\section{Resumen.}

La carrera de 400 metros en todas las pruebas de atletismo ha sido durante mucho tiempo un punto de atracción debido a las características biológicas que se llevan a cabo en este deporte. Esto, a su vez, ha generado mucho interés por parte de los entrenadores que buscan diversos recursos para conocer los factores limitantes del rendimiento, así como las características cardiovasculares más importantes de esta disciplina. Con el objetivo de: Describir los efectos de las

\footnotetext{
1 Universidad Técnica De Ambato. Pedagogía de la Actividad Física, Ambato, Ecuador. ga.villalba@uta.ed.ec. (D) https://orcid.org/0000-0001-5589-4927

2 Universidad Técnica De Ambato. Maestría en Actividad Física, Ambato, Ecuador. soledadrios451@gmail.com (D) https://orcid.org/0000-0002-9188-0378

${ }^{3}$ Universidad Nacional de Loja. Carrera de Pedagogía de la Actividad Física. Ecuador. vlady39quizhpe@gmail.com (D) https://orcid.org/0000-0002-0017-3097

${ }^{4}$ Universidad Nacional de Loja. Carrera de Pedagogía de la Actividad Física. Ecuador luis.valverde@unl.edu.ec (D) https://orcid.org/0000-0003-4810-6448
} 
em jovens corredores rasos de 400m. Um breve resumo da literatura disponível foi desenvolvido sobre as características do coração do jovem atleta. As revisões foram realizadas nas bases de dados Web of Science, Pubmed, EBSCO e Sports Discus até 15 de dezembro de 2020. Não foram estabelecidas restrições quanto ao idioma de busca. Os termos utilizados para a busca foram: Cardiolo * ou Cardiovas* e Jovens Atletas. Os critérios de inclusão considerados foram: (i) Definições, (ii) descobertas importantes, (iii) fatores influenciadores, (iv) estudos empíricos e (v) população entre 10 e 15 anos. Obtém-se como resultados recomendações para jovens atletas corredores de 400 metros com problemas do sistema cardiovascular.

Palavras-chave: Fatores de risco em atletas, fatores cardiovasculares em crianças atletas, Fisiologia de crianças atletas sobrecargas de entrenamiento en jóvenes corredores de poca profundidad de 400 $\mathrm{m}$. Se desarrolló un breve resumen de la literatura disponible sobre las características del corazón del atleta joven. Las revisiones se realizaron en las bases de datos de Web of Science, Pubmed, EBSCO y Sports Discus hasta el 15 de diciembre de 2020. No se establecieron restricciones en el idioma de búsqueda. Los términos utilizados para la búsqueda fueron: Cardiolo* o Cardiovas* y Atletas Jóvenes. Los criterios de inclusión considerados fueron: (i) Definiciones, (ii) hallazgos importantes, (iii) factores influyentes, (iv) estudios empíricos y (v) población entre 10 y 15 años. Como resultado, se obtienen recomendaciones para jóvenes corredores de 400 metros con problemas del sistema cardiovascular.

Palabras claves: Factores de riesgo en atletas, factores cardiovasculares en niños atletas, Fisiología de niños atletas

\section{Introdução}

O processo de treinamento e competição desportiva para a prova de $400 \mathrm{~m}$ em atletas adolescentes tende a ser relativamente homogêneo(Bombú et al., 2019), mas a variação nas características corporais, o tamanho do atleta, sua composição, bem como as proporções e maturação biológica podem ser consideráveis. (Valente-Dos-Santos et al., 2013) na definição do resultado. Eles requerem treinamento com cargas muito intensas onde intervém um componente estático moderado (20-50\% mvc) e com a presença de um componente dinâmico moderado, mas com peso voltado para o intensivo (40-70\% maxo2) (Gómez et al., 2020). Um treinamento com essas características apresenta fortes mobilizações da capacidade cardiovascular, o que gera uma série de alterações fisiológicas, hoje conhecidas como "coração de atleta" e que ao nível eletrocardiográfico apresentam uma série de padrões que devem ser reconhecida (Chandra et al., 2013) como um aumento nas catecolaminas circulantes, gerando uma resposta exagerada na pressão arterial, frequência cardíaca e contratilidade miocárdica, levando a um aumento no 
consumo de oxigênio do miocárdio , aumentando o risco de morte súbita (Gómez et al., 2020).

O desconhecimento desses padrões pode levar a falsos positivos e, por sua vez, a erros diagnósticos, realizando exames complementares realmente desnecessários (Chandra et al., 2013). A American Heart Association (AHA) e a European Society of Cardiology (ESC) mostraram documentos de consenso para a detecção de anormalidades em atletas (Semsarian et al., 2015). O ESC propõe como método de detecção, incluindo um histórico médico, um exame físico e um eletrocardiograma de 12 derivações em repouso. Em contraste, a AHA recomenda apenas uma história e exame físico. A razão para isso está no custo-benefício de sua realização (Semsarian et al., 2015).

Embora a anamnese seja considerada um método para determinar se há problemas cardiovasculares hereditários, para Gómez et al. (2020) o eletrocardiograma é o primeiro exame realizado na avaliação do atleta. Desde a publicação das primeiras recomendações para interpretação do eletrocardiograma em atletas, em 2005 (Corrado et al., 2005), ocorreram algumas modificações nos elementos encontrados que podem ser classificados em normais ou anormais. Reuniões de especialistas vêm sendo realizadas em diferentes partes do mundo com a análise e divulgação de novos critérios e concessões até chegar ao último publicado em 2017, no qual se inclui uma nova categoria, que são os chamados achados eletrocardiográficos limítrofes (Medrano Plana et al., 2019). Essa tecnologia é muito sensível para a detecção de cardiomiopatias hipertróficas, mas não muito específica, uma vez que 50\% dos atletas apresentam vestígios alterados, o que não implica em doença cardiovascular.

Por outro lado, o Ecocardiograma oferece a possibilidade de detectar anomalias mais profundas como hipertrofia nunca antes detectadas (Daher et al., 2019), algumas cardiopatias com características determinantes para suspensão da prática, mas é restrito pelo seu alto custo e acessibilidade para a maioria dos atletas, sendo enviado apenas em casos de detecção de problemas reais (Daher et al., 2019).

Existem várias investigações que estudam com a ecocardiografia diferentes parâmetros da função cardiovascular e sua variação interindividual associada ao tamanho corporal (Castanheira et al., 2014), esses indicadores ajudam a compreender o comportamento da maturação cardíaca de jovens atletas no que diz respeito à sua altura, que é considerada diretamente proporcional (Castanheira et al., 2014). Por sua vez, para Valente-Dos-Santos et al. (2014), A normalização da massa ventricular esquerda (MVE) para variação interindividual no tamanho do corpo é um tema central na biologia humana ... Este critério permite-nos coincidir com o critério de que, durante o período de crescimento da adolescência, variabilidade nos Descritores de tamanho corporal devem ser interpretados em combinação com a maturação biológica (Valente-Dos-Santos et al., 2014).

Entre as principais adaptações crônicas mais produzidas pelo treinamento com altas cargas para fins de alto rendimento em jovens está o aumento do diâmetro das câmaras 
cardíacas e da espessura das paredes. É justamente nesse indicador que o ventrículo esquerdo tem sido a estrutura do coração mais estudada (Hoogsteen et al., 2004).

Embora a cardiomiopatia hipertrófica ou cardiomiopatia arritmogênica do ventrículo direito tenham sido as doenças mais comuns (Basso et al., 2000), ainda existem poucas pesquisas sobre o comportamento desse ventrículo, devido à complexidade e magnitude morfológica do coração. e a variedade de anomalias congênitas das artérias coronárias (particularmente aquelas com origem sinusal incorreta) também consistentemente representam causas comuns de morte súbita relacionada ao exercício em jovens atletas (Basso et al., 2000).

Uma dessas malformações coronárias congênitas que deve ser constantemente revisada é a origem da artéria coronária no seio aórtico incorreto (que corre entre a aorta e o tronco pulmonar). Este parece ser o mais comum em jovens atletas treinados que morrem repentinamente. Na verdade, relatamos anteriormente que essas malformações estão associadas à morte súbita mais comumente em atletas competitivos do que em não atletas (Corrado et al., 1998; Maron, 2007; Panhuyzen-Goedkoop \& Verheugt, 2006).

Uma das evidências de morte súbita está associada à isquemia episódica ... porém, esta anomalia pode ser tratada cirurgicamente para sua correção, para a qual se revela a importância de poder detectá-la em tempo hábil durante as etapas do treinamento esportivo (Basso et al., 2000)

Para essas condições, o modelo americano (AHA): sugere que, se o estudo genético for positivo, mas o fenótipo não estiver presente, o atleta pode continuar com sua prática habitual. Em sua defesa, alegam que não há evidências de que haja motivos para separar esses atletas dos benefícios do esporte (Gómez et al., 2020). Já o modelo europeu (ESC): recomenda nos atletas com estudo genético positivo, mas sem fenótipo, abandonar o esporte competitivo, permitindo apenas o esporte recreativo. Para tanto, partem da hipótese de que o esporte competitivo pode desencadear o mecanismo que favorece o surgimento do fenótipo (Gómez et al., 2020).

Os óbitos são uma das causas que a literatura assume fortemente como o principal fator do overtraining, este critério é corroborado por um estudo (Harmon et al., 2016), que apresentou uma incidência geral de morte súbita de 0,98 / 100.000 atletas por ano. Diferenciada quanto ao sexo, nos homens a incidência foi de 0,99 / 100.000 atletas por ano, enquanto nas mulheres foi de 0,31 / 100.000 atletas por ano. O mesmo autor também estuda 53 mortes de atletas uni ᄀversitários, dos quais $41 \%$ correspondem a cardiomiopatia hipertrófica $(\mathrm{CMH}), 17 \%$ de causa inexplicada, $13 \%$ de miocardite, $7 \%$ de anomalia da artéria coronária (CAA), 5\% doença arterial coronariana (DAC), 3\% com myo cordis (CC), 3\% abuso de substâncias, 3\% aortopatias, 2\% doença arritmogênica miocárdica (ACM) e $2 \%$ insolação.

No mesmo ano, Finocchiaro et al. (2016) apresenta uma investigação sobre morte súbita no esporte, dividindo dois grupos para encontrar diferenças em termos de resultados. As principais causas no primeiro grupo $<18$ anos de idade foram: 56\% canalopatias, $16 \%$ cardiomiopatia hipertrófica $(\mathrm{CMH}), 11 \%$ anormalidades da artéria coronária (ACAC), 
$9 \%$ outras causas, $6 \%$ cardiomiopatia arritmogênica (ACA). ) e 2\% de miocardite. O segundo grupo com idades entre 18-35 anos também destacou canalopatias (44\%) como primeira causa, 23\% de cardiomiopatia hipertrófica (MCA), 14\% de cardiomiopatia arritmogênica (MCA), 10\% de outras causas, $4 \%$ de anomalia da artéria coronária (ACAC), $2 \%$ de miocardite e outra doença coronária de $2 \%$ prematuro (Finocchiaro et al., 2016).

As duas investigações anteriores apresentam um ponto de concordância com o que foi anteriormente afirmado por Suarez-Mier et al. (2013) em seu estudo sobre as causas de morte súbita em 81 atletas amadores com menos de 35 anos. De todos eles, 23\% tinham causa indeterminada, $18 \%$ cardiomiopatia hipertrófica, $15 \%$ cardiomiopatia arritmogênica, $13 \%$ doença arterial coronariana, $6 \%$ miocardite e $4 \%$ aortopatias.

Esses três estudos têm como fonte de citação o artigo apresentado por Basso et al. (2000) onde ECGs padrão de repouso de 12 derivações foram realizados em nove atletas, de 6 a 18 meses (média $11 \pm 3$ ) antes de sua morte súbita. Os padrões do eletrocardiograma (fig. 1), incluindo tensões da onda $T$, intervalos e morfologia, estavam dentro dos limites normais para cada um. No entanto, dois desses atletas isolaram complexos ventriculares prematuros.

Figura 1: Um jogador de futebol italiano de 15 anos com histórico de síncope por esforço

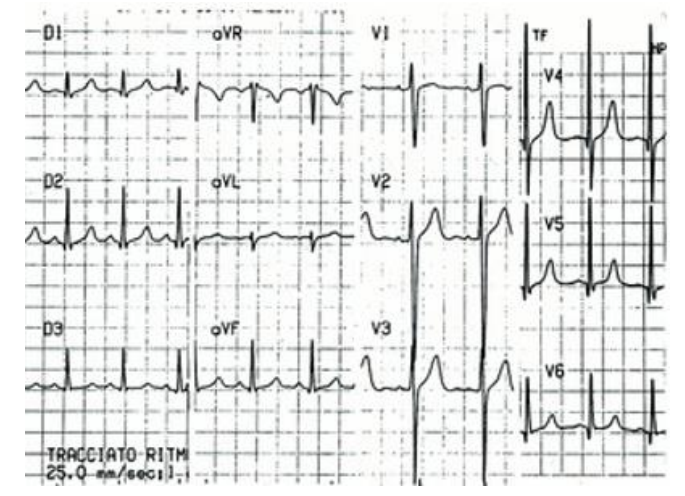

Fonte: Basso et al. (2000)

Este jogador de futebol, um ano antes da morte, morreu repentinamente enquanto corria durante a segunda metade de uma partida. (A) Um ECG de 12 derivações realizado 10 meses antes da morte, como parte da triagem de rotina antes da participação, está dentro dos limites normais. Basso et al. (2000).

A Figura 2 mostra este mesmo estudo Basso et al. (2000). a visão da raiz da aorta; A ACD normalmente se origina do seio aórtico direito (seta) e o TCE se origina anormalmente do seio direito com um descolamento em ângulo agudo, produzindo um lúmen em forma de fenda (ponta de seta). 
Figura 2: Visão da raiz aórtica do atleta

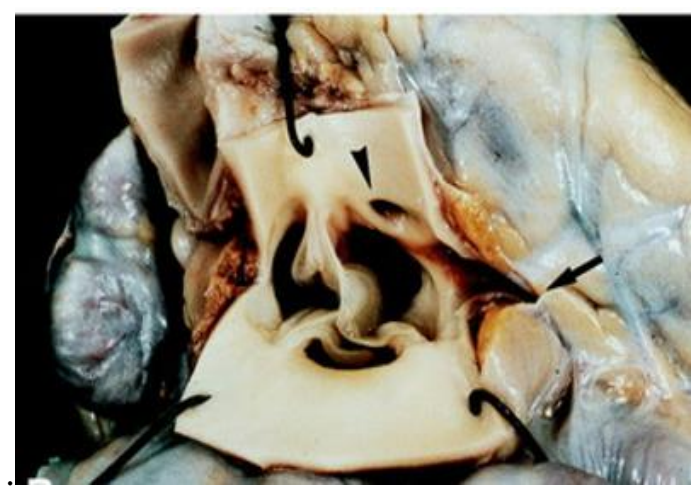

Fonte: Basso et al. (2000)

É muito importante observar que algumas doenças podem apresentar história negativa e ausência de sintomas ao exame físico em crianças. Em particular, doenças progressivas, como cardiomiopatia arritmogênica do ventrículo direito, que geralmente começa no final da adolescência, e doenças heterogêneas, como prolapso da válvula mitral, que pode aparecer em formas leves a graves (Fritsch et al., 2017), muitas vezes não apresentam sintomas em crianças, enfatizando a importância dos exames de acompanhamento (Elias Neto et al., 2019; Marcus et al., 2010; Platonov et al., 2016)

Por esse motivo, o exame físico deve ser considerado positivo se um sopro cardíaco qualquer grau diastólico e sistólico> 2/6, clique sistólico final, segundo simples ou amplamente dividido ou um som cardíaco fixo for detectado (Fritsch et al., 2017) uma vez que um pulso femoral lento ou atrasado, um ritmo cardíaco irregular ou leituras de pressão arterial elevada acima do percentil 95 também indicam um risco positivo (Neuhauser et al., 2011)

Uma das condições mais frequentes encontradas nas revisões realizadas são as arritmias cardiovasculares. Em relação a essa condição, existem critérios para trabalhá-los no esporte de diferentes formas. Para este trabalho citaremos que, a nosso ver, apresenta um embasamento adequado e que tem sido fortemente defendido nos últimos anos. Estas são as Recomendações para esportes em jovens com arritmias publicadas no artigo: Avaliação cardiovascular em jovens atletas ( Boccardo et al., 2000).

Tabela 1: Recomendações para esportes em jovens com arritmias

\begin{tabular}{ll}
\hline Arritmia & Recomendações \\
\hline Disfunção do nó sinusal & -Se a FC aumentar com o esforço, eles podem praticar esportes. Avaliação \\
& periódica. \\
& -Não há contra-indicação se não houver doença cardíaca estrutural. \\
& - Se a arritmia for assintomática, induzida por esforço e controlada com \\
Extrassístoles supraventriculares medicamentos, podem participar de competição. \\
e ventriculares isoladas & - Se a arritmia for recorrente / sintomática, deve ser tratada com RFA. \\
& -Depois de um período assintomático de 3 meses sem EPS de \\
& acompanhamento, você pode treinar para qualquer esporte. \\
\hline
\end{tabular}


Taquicardia supraventricular paroxística

Extrassístoles ventriculares polimórficas frequentes ou complexas (duplas e / ou triplas)

Taquicardia Ventricular Sustentada - Taquicardia Ventricular Não Sustentada Bloqueio AV de primeiro grau

Bloqueio AV congênito
O mesmo que pacientes com taquicardia ventricular.

Eles não devem participar até 6 meses após o tratamento (por qualquer método) e sem recorrência. Após esse período, se a ergometria estiver es podem competir.

Se houver doença estrutural, você só pode fazer atividades de baixa intensidade.

Se o paciente for assintomático, sem doença estrutural, e o bloqueio não piorar com o esforço, ele pode realizar atividade competitiva.

Se o paciente for assintomático, sem doença estrutural, complexo QRS estreito, FC de repouso> $50 \mathrm{bpm}$, que aumenta com o esforço, sem VE / VT pode competir. Após o implante de um marcapasso definitivo, você não poderá competir em esportes com alto contato físico.

Fuente: (Boccardo et al., 2000).

\section{Métodos}

\section{Identificação dos estudos.}

Foi desenvolvida uma revisão sistemática da literatura quantitativa. Dois revisores independentes contribuíram separadamente entre 10 e 13 de Novembro de 2020, utilizando dados recolhidos entre 2001 e 2020. As bases de dados Web of Sience, EBSCO, PSYCHINFO e ERIC foram utilizadas para garantir desde o início a qualidade das análises efectuadas. Os termos utilizados para a busca foram: Cardiolo * ou Cardiovas * e Jovens Atletas. Os critérios de inclusão e exclusão foram utilizados tal como apresentados na tabela 1 .

Tabla.1: Critérios de inclusão y Critérios de exclusão do estudo.

\begin{tabular}{llll}
\hline \multicolumn{2}{l}{ Critérios de inclusão } & \multicolumn{2}{l}{ Critérios de exclusão. } \\
\hline I. Definiciones, textos escritos & I. & Obras publicadas em congreso. \\
inteiramente em inglês. & II. Revisores independentes. \\
II. Descobertas importantes e perspectivas & III. & $\begin{array}{l}\text { Estudos que no incluíam sexo. } \\
\text { exploradas. }\end{array}$ & IV. Teses e monografias também foram \\
III. Fatores influenciadores Publicado em & excluídas. \\
estudos empíricos. & & \\
IV. Amostragem com atletas entre 10 y 15 & & \\
anos. &
\end{tabular}

Fonte: Elaboração própria para o trabalho de investigação.

\section{Organização dos dados}

A organização da análise dos resultados foi organizada de acordo com os dados para o controlo do desempenho disponíveis na literatura de acordo com o modelo PRISMA disponível na figura 1, onde foram identificados um total de 267 artigos. Um total de 32 papéis foram retirados como duplicados, deixando 235 na fase de identificação. Após uma triangulação de critérios, um total de 200 referências foram excluídas, restando 35 para a análise do texto completo. Nesta fase, foram excluídas 18 referências que preenchiam os critérios de inclusão. 
Fluxograma de pesquisa PRISMA

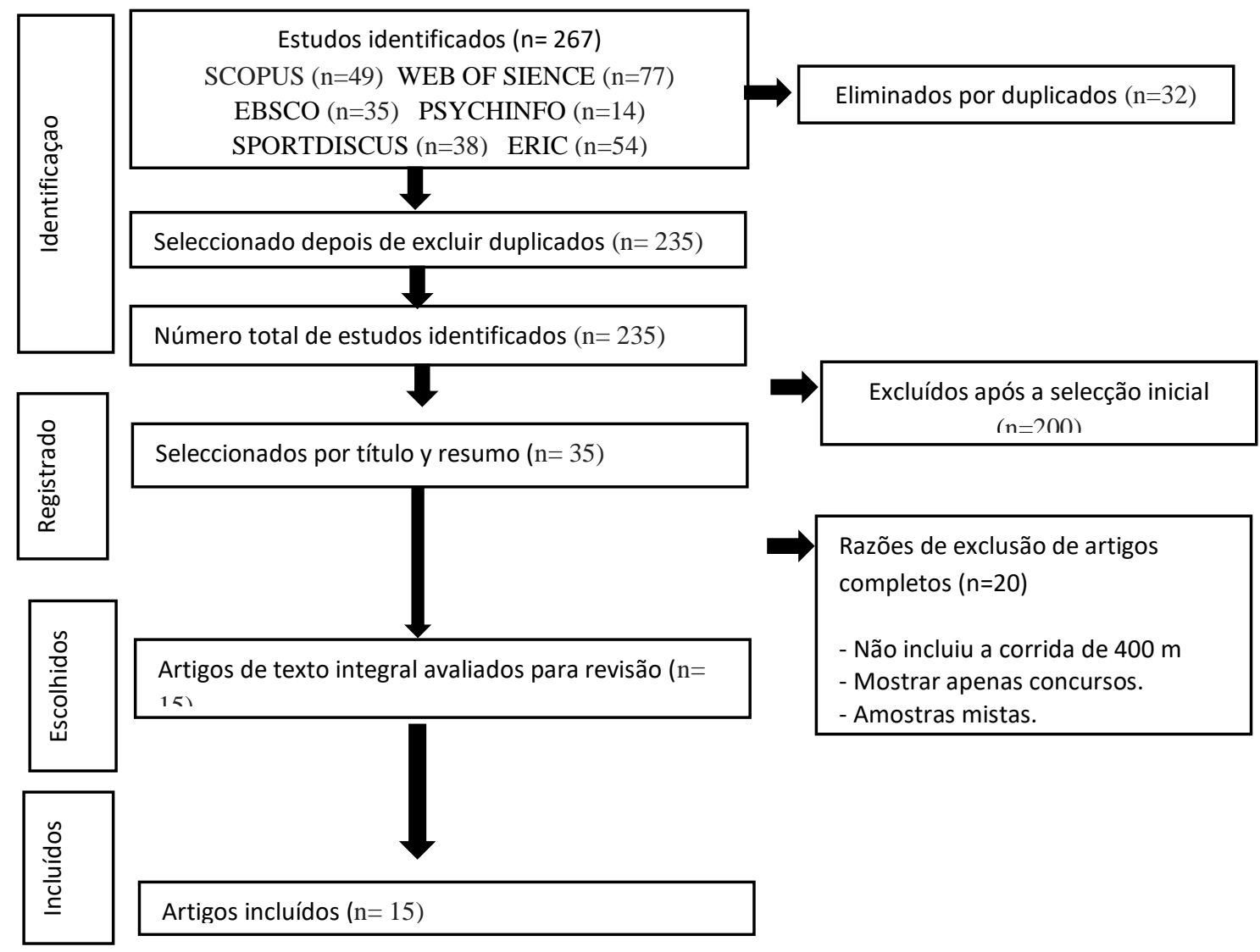

Fonte: Elaboração própria para o trabalho de investigação.

\section{Discussão}

Atletas com defeito do septo atrial pequeno não tratado, pressão pulmonar normal, sem disfunção ventricular ou arritmias significativas (Repáraz et al., 2010), podem participar com avaliações anuais.

Atletas com hipertensão pulmonar só podem participar desse esporte com baixa intensidade, mas não para fins competitivos (Dios et al., 2013).

Atletas com doença pulmonar obstrutiva associada à cianose e shunt da direita para a esquerda não podem praticar esse desporte.

Atletas bem-sucedidos sem sinais de hipertensão pulmonar, arritmias significativas, bloqueios AV de segundo ou terceiro grau ou disfunções cardíacas óbvias podem participar com avaliações anuais ( 3 a 6 meses após a correção). Na presença de qualquer uma das variáveis descritas, deve-se considerar o seu tratamento e participação em competições, limitando-se a treinamentos de intensidade baixa a moderada, conforme avaliação. 
Atletas assintomáticos, sem história de aneurisma do septo atrial, com achado de pequeno aneurisma do septo atrial com ou sem forame oval patente podem participar se apresentarem teste ergométrico máximo normal, sem arritmias significativas.

Atletas com comunicação intraventricular (CIV) pequena não tratada, sem dilatação das cavidades ou sobrecargas de volume ou pressão do VD podem participar desta disciplina.

Atletas com CIV moderada ou grande, com baixa resistência vascular pulmonar e relação fluxo pulmonar / fluxo sistêmico maior ou igual a 2 e resistência pulmonar menor que 3 $\mathrm{U} / \mathrm{m} 2$ podem participar de desportes de intensidade dinâmica baixa / moderada e estática baixa, mas não esta disciplina devido ao seu componente de alta intensidade ao programar a correção do defeito por cateterismo ou cirurgia (Michels et al., 1992)

Atletas com fechamento de CIV com sucesso (sem defeito residual ou defeito mínimo) e sem sinais de hipertensão pulmonar, arritmias significativas ou disfunções cardíacas residuais podem participar deste desporte ( 3 a 6 meses após a correção).

Atletas com canal arterial pequeno (PCA), cavidades normais e sem hipertensão pulmonar (comum em PCA pequeno) podem participar desse desporte com controles cardiovasculares frequentes.

Em atletas com média a grande resolução de PCA por cateterismo é recomendado antes de autorizar exercícios competitivos.

Atletas com grande PCA e hipertensão pulmonar grave e cianose devem evitar participar dessas corridas (Castro Beiras et al., 2003)

Atletas assintomáticos com estenose valvular pulmonar (DVP), gradiente $<40 \mathrm{~mm} \mathrm{Hg}$, função ventricular direita normal e nenhuma (ou apenas leve) dilatação do VD podem participar deste esporte. Recomenda-se considerá-los "aptos transitórios" com reavaliações anuais. (Hershberger \& Siegfried, 2011)

Atletas assintomáticos com estenose do orifício aórtico congênita leve (ASS) podem participar se tiverem um ECG de repouso normal e teste de esforço sem arritmias significativas, com tolerância adequada ao exercício.

Atletas assintomáticos com coarctação de aorta CoAo leve, gradientes de pressão sistólica $<20 \mathrm{~mm} \mathrm{Hg}$ entre a parte superior e inferior do corpo, nenhuma hipertensão arterial na parte superior do corpo e nenhuma dilatação significativa da raiz da aorta (escore z de 3,0 ou menos), com valores de pressão arterial normais para a idade durante o teste ergométrico, podem praticar a prática desportiva.

Atletas com insuficiência pulmonar grave ou hipertensão ventricular direita $(\geq 50 \%$ da pressão sistêmica) ou arritmias significativas ou shunt persistente só podiam participar deste desporte. 


\section{Conclusões}

- Levando em consideração todo o acúmulo de condições previamente expostas, deve-se ter um maior controle sobre o processo de treinamento esportivo desses jovens corredores de 400 metros e o controle de sua carga de treinamento.

- Em atletas que ainda estão em processo de maturação, deve-se fazer anamnese ou exame físico para descartar que este possa ser acometido por algum tipo de cardiopatia genética. Este estudo não deve estar diretamente associado à patologia de que possamos suspeitar, mas deve ser orientado de forma geral para a descarga de outras anomalias congênitas ou genéticas.

- A realização de estudos genéticos em curto ou médio prazo pode prevenir a morte cardiovascular no desporte e embora ainda haja debates sobre se o atleta deve praticar desporte no caso de apresentar patologias genéticas positivas, se atualmente está claro para você, quais são as marcadores cardiovasculares que impedem drasticamente qualquer tipo de atividade física intensa.

- A inclusão de novas tecnologias na genética está mudando as estratégias de estudo das doenças de origem genética. Em um futuro próximo, o ultra sequenciamento reduzirá custos e acelerará o processo. Graças a isso, os estudos genéticos não estarão tão avançados como até agora, quando se buscava um diagnóstico.

\section{Referências bibliográficas.}

Basso, C., Maron, B. J., Corrado, D., \& Thiene, G. (2000). Clinical profile of congenital coronary artery anomalies with origin from the wrong aortic sinus leading to sudden death in young competitive athletes. Journal of the American College of Cardiology, 35(6), 1493-1501.

Boccardo, D., Tibaldi, M., \& Coll, M. (2000). La evaluación cardiovascular en jóvenes deportistas. Revista Costarricense de Cardiología, 2, 35-45. http://www.scielo.sa.cr/scielo.php?script=sci_arttext\&pid=S1409$41422000000200007 \&$ nrm $=$ iso

Bombú, R. M., Toro, V. H., Ortiz, P. O., \& Acosta, W. C. (2019). Contribuciones al control físico-pedagógico del rendimiento deportivo de los corredores de $400 \mathrm{~m}$ planos. Ciencia Digital, 3(2.5), 32-45.

Castanheira, J., Valente-dos-Santos, J., Duarte, J., Vaz, V., Figueiredo, A. J., Leite, N., Cyrino, E. S., \& Coelho-e-Silva, M. J. (2014). Left ventricular morphology in adolescents: comparison between athletes and non-athletes. Revista Brasileira de Medicina do Esporte, 20(6), 480-485.

Castro Beiras, A., Monserrat, L., \& Hermida, M. (2003). [Familial dilated cardiomyopathy: current status and clinical benefits of basic research]. Rev Esp Cardiol, 56 Suppl 1, 7-12. (Miocardiopatía dilatada familiar: situación actual y beneficios clínicos de la investigación básica.) 
Chandra, N., Bastiaenen, R., Papadakis, M., \& Sharma, S. (2013). Sudden cardiac death in young athletes: practical challenges and diagnostic dilemmas. Journal of the American College of Cardiology, 61(10), 1027-1040.

Corrado, D., Basso, C., Schiavon, M., \& Thiene, G. (1998, Aug 6). Screening for hypertrophic cardiomyopathy in young athletes. N Engl J Med, 339(6), 364-369. https://doi.org/10.1056/NEJM199808063390602

Corrado, D., Pelliccia, A., Bjornstad, H. H., Vanhees, L., Biffi, A., Borjesson, M., Panhuyzen-Goedkoop, N., Deligiannis, A., Solberg, E., Dugmore, D., Mellwig, K. P., Assanelli, D., Delise, P., van-Buuren, F., Anastasakis, A., Heidbuchel, H., Hoffmann, E., Fagard, R., Priori, S. G., Basso, C., Arbustini, E., BlomstromLundqvist, C., McKenna, W. J., Thiene, G., Study Group of Sport Cardiology of the Working Group of Cardiac, R., Exercise, P., the Working Group of, M., \& Pericardial Diseases of the European Society of, C. (2005, Mar). Cardiovascular pre-participation screening of young competitive athletes for prevention of sudden death: proposal for a common European protocol. Consensus Statement of the Study Group of Sport Cardiology of the Working Group of Cardiac Rehabilitation and Exercise Physiology and the Working Group of Myocardial and Pericardial Diseases of the European Society of Cardiology. Eur Heart J, 26(5), 516-524. https://doi.org/10.1093/eurheartj/ehi108

Daher, G., Hassanieh, I., Malhotra, N., \& Alderson, L. (2019, Oct 22). Acute Decompensated Heart Failure Secondary to Exogenous Triiodothyronine Use in a Young Non-athlete Weightlifter. Cureus, 11(10), e5964. https://doi.org/10.7759/cureus.5964

Dios, A. d., Naya, E. G., \& Tortorella, R. (2013). Recomendaciones para la Participación en Deportes Competitivos en Personas con Anormalidades Cardiovasculares Sociedad argentina de cardiología Área de normatizacioneS y conSenSoS.

Elias Neto, J., Tonet, J., Frank, R., \& Fontaine, G. (2019, Jan). Arrhythmogenic Right Ventricular Cardiomyopathy/Dysplasia (ARVC/D) - What We Have Learned after 40 Years of the Diagnosis of This Clinical Entity. Arq Bras Cardiol, 112(1), 91-103. https://doi.org/10.5935/abc.20180266

Finocchiaro, G., Papadakis, M., Robertus, J. L., Dhutia, H., Steriotis, A. K., Tome, M., Mellor, G., Merghani, A., Malhotra, A., Behr, E., Sharma, S., \& Sheppard, M. N. (2016, May 10). Etiology of Sudden Death in Sports: Insights From a United Kingdom Regional Registry. J Am Coll Cardiol, 67(18), 2108-2115. https://doi.org/10.1016/j.jacc.2016.02.062

Fritsch, P., Pozza, R. D., Ehringer-Schetitska, D., Jokinen, E., Herceg, V., Hidvegi, E., Oberhoffer, R., \& Petropoulos, A. (2017, Nov). Cardiovascular pre-participation screening in young athletes: Recommendations of the Association of European Paediatric Cardiology - CORRIGENDUM. Cardiol Young, 27(9), 1661. https://doi.org/10.1017/S1047951117001986 
Gómez, F. M. V., García, D. F., Salgado, J. G., Dias, A., García-Iglesias, J. J., \& Frutos, C. R. (2020). Prevención de la muerte súbita cardiaca en el deportista joven desde la perspectiva enfermera. RqR Enfermería Comunitaria, 8(1), 36-45.

Harmon, K. G., Asif, I. M., Maleszewski, J. J., Owens, D. S., Prutkin, J. M., Salerno, J. C., Zigman, M. L., Ellenbogen, R., Rao, A. L., Ackerman, M. J., \& Drezner, J. A. (2016, Nov). Incidence and Etiology of Sudden Cardiac Arrest and Death in High School Athletes in the United States. Mayo Clin Proc, 91(11), 1493-1502. https://doi.org/10.1016/j.mayocp.2016.07.021

Hershberger, R. E., \& Siegfried, J. D. (2011, Apr 19). Update 2011: clinical and genetic issues in familial dilated cardiomyopathy. J Am Coll Cardiol, 57(16), 1641-1649. https://doi.org/10.1016/j.jacc.2011.01.015

Hoogsteen, J., Hoogeveen, A., Schaffers, H., Wijn, P. F., van Hemel, N. M., \& van der Wall, E. E. (2004, Feb). Myocardial adaptation in different endurance sports: an echocardiographic study. Int $J$ Cardiovasc Imaging, 20(1), 19-26. https://doi.org/10.1023/b:caim.0000013160.79903.19

Marcus, F. I., McKenna, W. J., Sherrill, D., Basso, C., Bauce, B., Bluemke, D. A., Calkins, H., Corrado, D., Cox, M. G., Daubert, J. P., Fontaine, G., Gear, K., Hauer, R., Nava, A., Picard, M. H., Protonotarios, N., Saffitz, J. E., Sanborn, D. M., Steinberg, J. S., Tandri, H., Thiene, G., Towbin, J. A., Tsatsopoulou, A., Wichter, T., \& Zareba, W. (2010, Apr 6). Diagnosis of arrhythmogenic right ventricular cardiomyopathy/dysplasia: proposed modification of the task force criteria. Circulation, $121(13)$,

1533-1541. https://doi.org/10.1161/CIRCULATIONAHA.108.840827

Maron, B. J. (2007, Aug). Hypertrophic cardiomyopathy and other causes of sudden cardiac death in young competitive athletes, with considerations for preparticipation screening and criteria for disqualification. Cardiol Clin, 25(3), 399-414, vi. https://doi.org/10.1016/j.ccl.2007.07.006

Medrano Plana, Y., Castillo Marcillo, Á. R., Lugo Morales, A. M., \& Arévalo Andrade, M. A. (2019). Alteraciones electrocardiográficas en jóvenes atletas de alto rendimiento. CorSalud, 296-301. http://scielo.sld.cu/scielo.php?script=sci_arttext\&pid=S2078$71702019000400296 \&$ nrm $=$ iso

Michels, V. V., Moll, P. P., Miller, F. A., Tajik, A. J., Chu, J. S., Driscoll, D. J., Burnett, J. C., Rodeheffer, R. J., Chesebro, J. H., \& Tazelaar, H. D. (1992, Jan 9). The frequency of familial dilated cardiomyopathy in a series of patients with idiopathic dilated cardiomyopathy. $N$ Engl J Med, 326(2), 77-82. https://doi.org/10.1056/NEJM199201093260201

Neuhauser, H. K., Thamm, M., Ellert, U., Hense, H. W., \& Rosario, A. S. (2011). Blood pressure percentiles by age and height from nonoverweight children and adolescents in Germany. Pediatrics, 127(4), e978-e988. 
Panhuyzen-Goedkoop, N. M., \& Verheugt, F. W. (2006, Sep). Sudden cardiac death due to hypertrophic cardiomyopathy can be reduced by pre-participation cardiovascular screening in young athletes. Eur Heart J, 27(18), 2152-2153. https://doi.org/10.1093/eurheartj/ehl171

Platonov, P. G., Calkins, H., Hauer, R. N., Corrado, D., Svendsen, J. H., Wichter, T., Biernacka, E. K., Saguner, A. M., Te Riele, A. S., \& Zareba, W. (2016, Jan). High interobserver variability in the assessment of epsilon waves: Implications for diagnosis of arrhythmogenic right ventricular cardiomyopathy/dysplasia. Heart Rhythm, 13(1), 208-216. https://doi.org/10.1016/j.hrthm.2015.08.031

Repáraz, J. M., Pesenti, M. G., Ormella, M., Vilas, M. S., \& Ramos, M. H. (2010). MUERTE SUBITA EN ATLETAS JOVENES. Revista Española de Cardiología Suplementos, 10, 78A-84A.

Semsarian, C., Sweeting, J., \& Ackerman, M. J. (2015, Mar 18). Sudden cardiac death in athletes. BMJ, 350, h1218. https://doi.org/10.1136/bmj.h1218

Suarez-Mier, M. P., Aguilera, B., Mosquera, R. M., \& Sanchez-de-Leon, M. S. (2013, Mar 10). Pathology of sudden death during recreational sports in Spain. Forensic Sci Int, 226(1-3), 188-196. https://doi.org/10.1016/j.forsciint.2013.01.016

Thomas, J., \& Harden, A. (2008). Methods for the thematic synthesis of qualitative research in systematic reviews. BMC medical research methodology, 8(1), 45.

Valente-Dos-Santos, J., Coelho-e-Silva, M. J., Vaz, V., Figueiredo, A. J., Castanheira, J., Leite, N., Sherar, L. B., Baxter-Jones, A., Elferink-Gemser, M. T., \& Malina, R. M. (2013, Jul). Ventricular mass in relation to body size, composition, and skeletal age in adolescent athletes. Clin J Sport Med, 23(4), 293-299. https://doi.org/10.1097/JSM.0b013e318280ac63

Valente-Dos-Santos, J., Coelho, E. S. M. J., Ferraz, A., Castanheira, J., Ronque, E. R., Sherar, L. B., Elferink-Gemser, M. T., \& Malina, R. M. (2014, Sep-Oct). Scaling left ventricular mass in adolescent boys aged 11-15 years. Ann Hum Biol, 41(5), 465-468. https://doi.org/10.3109/03014460.2013.866694

\section{Clencia}




\section{PARA CITAR EL ARTÍCULO INDEXADO.}

Lima Rodríguez, AL., Hernández Barrenechea, AL., Martínez González, LE., \& Laguardia Alfonso, YL. (2021). La educación alimentaria y nutricional desde el proceso de enseñanza-aprendizaje de la Antomía y Fisiología Humana. Revista electrónica Anatomía Digital 3(2), 78-97. Recuperado desde: http://anatomiadigital.org/revistacienciadigital2/index.php/AnatomiaDigital/article/view $\underline{1263 / 567}$

\section{Ciencia \\ LDigital}

El artículo que se publica es de exclusiva responsabilidad de los autores y no necesariamente reflejan el pensamiento de la Revista Anatomía Digital.

El artículo queda en propiedad de la revista y, por tanto, su publicación parcial y/o total en otro medio tiene que ser autorizado por el director de la Revista Anatomía Digital.
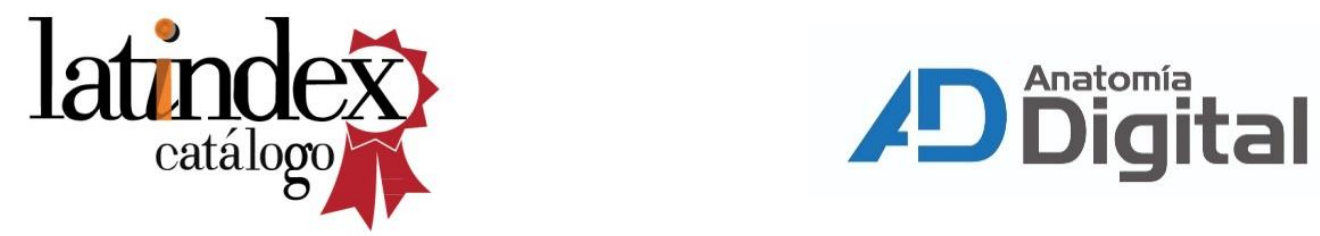